Research Article

\title{
Simulation and Visualization of Flows Laden with Cylindrical Nanoparticles in a Mixing Layer
}

\author{
Wenqian Lin (i) ${ }^{1}$ and Peijie Zhang ${ }^{2}$ \\ ${ }^{1}$ College of Computer Science and Technology, Zhejiang University, Hangzhou 310027, China \\ ${ }^{2}$ Department of Mechanics, Zhejiang University, Hangzhou 310027, China \\ Correspondence should be addressed to Wenqian Lin; linwq@zju.edu.cn
}

Received 31 January 2018; Accepted 27 February 2018; Published 5 April 2018

Academic Editor: Mingzhou Yu

Copyright (c) 2018 Wenqian Lin and Peijie Zhang. This is an open access article distributed under the Creative Commons Attribution License, which permits unrestricted use, distribution, and reproduction in any medium, provided the original work is properly cited.

\begin{abstract}
The motion of cylindrical particles in a mixing layer is studied using the pseudospectral method and discrete particle model. The effect of the Stokes number and particle aspect ratio on the mixing and orientation distribution of cylindrical particles is analyzed. The results show that the rollup of mixing layer drives the particles to the edge of the vortex by centrifugal force. The cylindrical particles with the small Stokes number almost follow fluid streamlines and are mixed thoroughly, while those with the large Stokes number, centrifugalized and accumulated at the edge of the vortex, are poorly mixed. The mixing degree of particles becomes worse as the particle aspect ratio increases. The cylindrical particles would change their orientation under two torques and rotate around their axis of revolution aligned to the vorticity direction when the shear rate is low, while aligning on the flow-gradient plane beyond a critical shear rate value. More particles are oriented with the flow direction, and this phenomenon becomes more obvious with the decrease of the Stokes number and particle aspect ratio.
\end{abstract}

\section{Introduction}

Mixing of nanoparticles in a flow was found in many applications and has been a subject of interest during the recent twenty years. The mixing of nanoparticles affects the property of the flow and the behaviors of the final production. Therefore, understanding deeply such a flow is important to design a novel technology for nanoparticle manipulation.

Some efforts have been put into the numerical simulation and visualization of nanoparticles. Singh et al. [1] deposited $\mathrm{In}_{2} \mathrm{O}_{3}$ and $\mathrm{In}_{2} \mathrm{O}_{3}$ : Ag nanoparticle layers and indicated the presence of $\mathrm{Ag}_{2} \mathrm{O}$ and $\mathrm{Ag}$ in air- and vacuumannealed samples, respectively. Aruna et al. [2] reported the modifications in $\mathrm{CO}$ sensing of $\mathrm{SnOx}$ nanoparticle layers by using Pd nanoparticles; they found that the homogeneously mixed nanoparticle layers show capability between $\mathrm{CO}$ and ethanol as a manifestation of the dual conductance response. Hsiao et al. [3] used mixed PSS-DTMA Langmuir layers to incorporate with silver precursors from the subphase and transferred them onto mica substrates; they showed that enhancing the DTMA (+) in the mixed PSS-DTMA system would increase the hydrophobic property of the complexes. Finally, they inferred that the polyelectrolyte-surfactant template can offer a potential of designing structures of polyelectrolyte-nanoparticle materials. Liggieri et al. [4] utilized different techniques to measure the dilational viscoelasticity in a wide frequency range and used these data to provide qualitative and quantitative information about structural behaviors of complex mixed layers. Xie et al. [5] simulated numerically the impact coherent structure on the Brownian coagulation of particles in a mixing layer, and it is found that the number density of nanoparticles decreases gradually as the flow evolves, while the particle average volume increases. The impact of fluid advection on particle coagulation is small in the regions far away from the eddy structure. The particle coagulation in the eddy core has a wave-like distribution. Yazhgur et al. [6] studied the adsorption films of silica nanoparticles modified by a cationic surfactant at the air-water interface. They divided the 
whole surfactant concentration range into four regions characterized by different surface rheological behaviors. Kim et al. [7] studied the optical, electrical, and morphological behaviors of the $\mathrm{Ga}_{2} \mathrm{O}_{3} \mathrm{NP} / \mathrm{SWNT}$ layers by enhancing the thickness of SWNTs. Murfield and Garrick [8] performed numerical simulations of nanoparticle nucleation in turbulent wakes and showed that nucleation initially occurs in the shear layers where molecular transport dominates and across the span of the wake. Fager and Garrick [9] presented the results of direct numerical simulation of zinc nanoparticle nucleation in a turbulent round jet; they indicated that particle nucleation occurs along the outer region of the jet, and the regions over which nucleation occurs increase significantly after collapse of the jet potential core. Guzman et al. [10] carried out measurements of the dilational viscoelastic modulus against the frequency by the oscillatory barrier method at different degrees of compression of the monolayer in order to deepen the understanding of the impacts of nanoparticles on the interfacial properties of biosystems. Orsi et al. [11] studied the interfacial dynamics of a 2D self-organized mixed layer consisted of silica nanoparticles interacting with phospholipid monolayers at the air-water interface and found a dynamical transition from the Brownian diffusion to an arrested state. Garrick [12] showed that, in the proximal region of the jet, condensation is the dominant mechanism; however, once the jet potential core collapses and turbulent mixing begins, coagulation is the dominant mechanism. Kerli and Alver [13] investigated the mixture of $\mathrm{ZnO}$ and $\mathrm{NiO}$ effect on the solar cell and observed that the solar cells made with $\mathrm{ZnO}$ have the highest performance with the efficiency of $0.542 \%$.

The researches available in the literature are mainly concerned with spherical particles as shown above. However, many particles in the practical usage are nonspherical among which the cylinder particle is a typical example. Compared with the spherical particle, the mixing of cylindrical particles in a flow is more complicated because the change of particle orientation is coupled with the translation motion. Understanding the property of cylindrical particles in the mixing layer is a great importance in the optimization of production processes. As far as we know, the motion and mixing behaviors of cylindrical nanoparticles in the mixing layer have not been reported in the literatures yet. Therefore, the objective of the present paper is to explore the impact of the Stokes number and particle aspect ratio on the mixing property and orientation distributions of cylindrical particles in a mixing layer.

\section{Flow and Basic Equations}

2.1. Mixing Layer Flow and Fluid Equation. A mixing layer consisting of two streams and a cylindrical particle are shown in Figure 1 where velocities $U_{1}$ and $U_{2}$ are different. In the mixing layer, the hydrodynamic instabilities will happen, which leads to the flow to roll up and form coherent vortex and even pairing of the vortices.

Dilute cylindrical particle suspension is studied here, that is, the effect of the cylindrical particle on the fluid can be
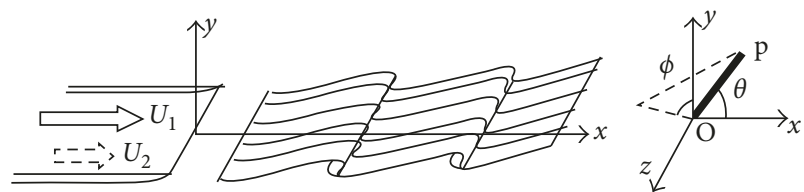

Figure 1: Mixing layer flow and cylindrical particle.

ignored; thus, the governing equations are the following continuity and momentum equations:

$$
\begin{aligned}
\nabla \cdot u & =0, \\
\frac{\partial u}{\partial t}+\nabla\left(p+\frac{|u|^{2}}{2}\right) & =u \times \omega+\frac{\nabla^{2} u}{\operatorname{Re}},
\end{aligned}
$$

where $u$ and $p$ are the fluid velocity and pressure, respectively; $\omega$ is the vorticity; and Re is the Reynolds number.

2.2. Equation for Cylindrical Particles. The size of a nonBrownian and rigid cylindrical particle is smaller than the characteristic scale of the flow field. The motion of particles is caused by force $F$ and torque $T$ exerted by the fluid:

$$
\begin{gathered}
m_{\mathrm{p}} \frac{d u_{\mathrm{p}}}{d t}=F=\int_{-l}^{l} f(s) d s, \\
I \cdot \frac{d \Omega}{d t}+\Omega \times I \cdot \Omega=T=\int_{-l}^{l} s \mathbf{p} \times f(s) d s,
\end{gathered}
$$

where $m_{\mathrm{p}}, u_{\mathrm{p}}$, and $\Omega$ are the particle mass, velocity, and angular velocity, respectively; $I$ is the particle moment of inertia; $f(s)$ is the force distribution along the particle axis; $s$ is the distance from any point to the particle center; $l$ is the half length of the particle; and $\mathbf{p}$ is the orientation vector of the particle.

Equation (3) can be nondimensionalized with the scale of the flow field as

$$
\begin{aligned}
& \frac{d u_{\mathrm{p}}}{d t}=\frac{1}{\mathrm{St}} \int_{-1}^{1} f(s) d s, \\
& \frac{d \Omega_{x}}{d t}=\frac{3}{a \mathrm{St}}\left(\frac{4 \mathrm{St}}{\operatorname{Re} \rho^{*}}\right)^{-1 / 2} T_{x}+\Omega_{y} \Omega_{z}, \\
& \frac{d \Omega_{y}}{d t}=\frac{3}{a \mathrm{St}}\left(\frac{4 \mathrm{St}}{\operatorname{Re} \rho^{*}}\right)^{-1 / 2} T_{y}-\Omega_{z} \Omega_{x}, \\
& \frac{d \Omega_{z}}{d t}=\frac{2 a}{\mathrm{St}}\left(\frac{4 \mathrm{St}}{\operatorname{Re} \rho^{*}}\right)^{-1 / 2} T_{z},
\end{aligned}
$$

where $a$ is the particle aspect ratio, St is the Stokes number ( $\mathrm{St}=\rho_{p} r^{2} U_{0} / 2 \mu \theta_{0}$, in which $\rho_{\mathrm{p}}$ is the particle density, $r$ is the particle radius, $U_{0}=U_{1}-U_{2}$, and $\theta_{0}$ is the initial momentum thickness of the mixing layer), and $\rho^{*}$ is the particle-to-fluid density ratio. 


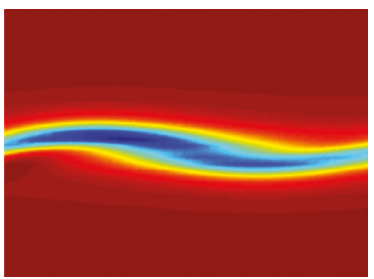

(a)

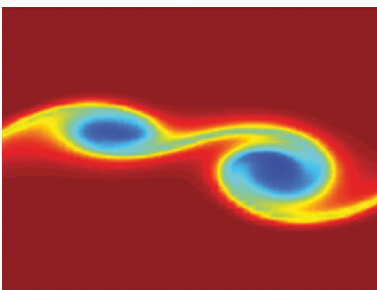

(a)

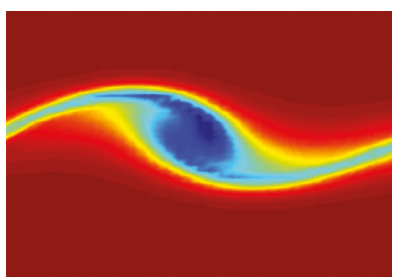

(b)

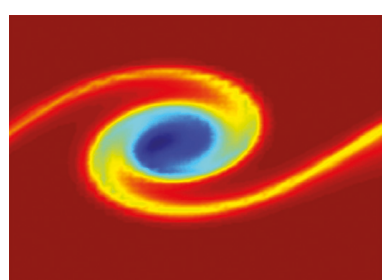

(c)

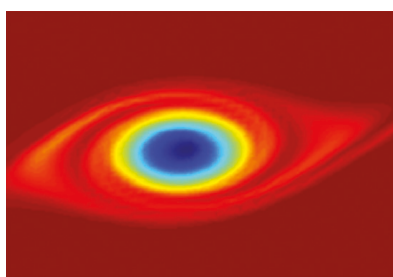

(d)

Figure 2: Rollup of the mixing layer.

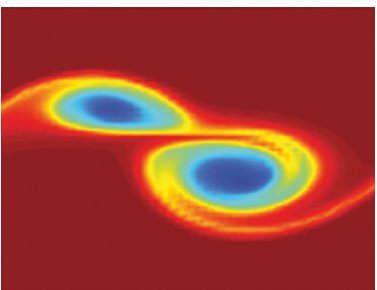

(b)

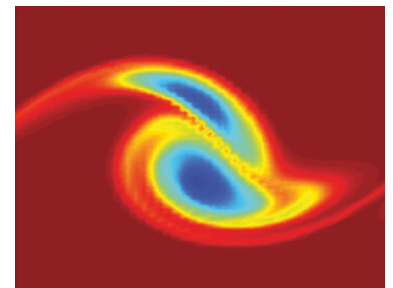

(c)

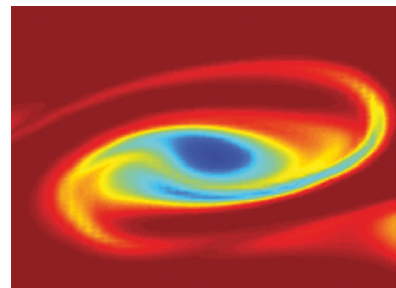

(d)

FIgURE 3: Vortex pairing.

\section{Numerical Simulation}

3.1. Initial Condition of the Flow. For the temporally evolving mixing layer, the initial condition of the flow consists of two parts:

(1) The velocity profile with hyperbolic-tangent form of the base flow:

$$
U=0.5 \tanh (y) .
$$

(2) The lower wavenumber disturbances with stream function:

$$
\psi(x, y)=A_{1} \mathrm{R}\left[\varphi_{1}(y) e^{i a x}\right]+A_{2} \mathrm{R}\left[\varphi_{2}(y) e^{i(a / 2) x}\right],
$$

where $\psi$ is the stream function; $A_{1}$ and $A_{2}$ are the amplitude of the fundamental and its subharmonic modes, respectively, and we set $A_{1}=0.1$ and $A_{2}=0$ and $A_{1}=0.1$ and $A_{2}=0.06$ for the process of vortex rollup and vortex pairing; $\mathrm{R}$ means the real part for a complex; $\varphi_{1}(y)$ and $\varphi_{2}(y)$ are the eigenfunctions for the fundamental and subharmonic modes, respectively; and $\alpha$ is the fundamental wavenumber and is set to be 0.4446 [14].

3.2. Boundary Condition. The periodic boundary conditions in the streamwise $(x)$ and transverse $(y)$ directions are imposed by introducing an image flow far enough from the mixing layer center because all the perturbations vanish as $y \rightarrow \infty$. Therefore, the Fourier spectral method can be exerted in both directions, and fast Fourier transformation can be used. The periodic $L_{1}$ and $L_{2}$ in the streamwise and transverse directions are, respectively, taken to be $2 \pi / r$ and
28 for the vortex rollup, and both are doubled for the vortex pairing.

3.3. Computational Approach. Equations (1) and (2) are numerically solved using the pseudospectral method. The Adams-Bashforth scheme is used for the nonlinear term, and the implicit Crank-Nicolson scheme is used for other terms. The time step is set to be 0.03 . The collection points in the streamwise and transverse directions are taken to be 128 and 256, respectively.

Equations (4) and (5) are solved with the fourth-order Runge-Kutta method for 400 cylindrical particles. These particles are distributed and oriented homogeneously in the upper layer of the flow as shown in Figure 1. The time step of the integration is small enough to guarantee a stable trajectory of the particles.

\section{Results and Discussions}

4.1. Visualization of Vortex Rolling Up and Vortex Pairing. The Kelvin-Helmholtz instability makes the mixing layer roll up and forms the large-scale vortex as shown in Figure 2, where the center of the vortex is called the center point and the middle point of two center points is called the saddle point. The flow can be divided into the inhomogeneous region and the homogeneous region. The streamlines connecting two saddle points are defined as the demarcation lines; the region encompassed by the streamlines, that is, vortex core, is considered as the inhomogeneous region, and the other region is called the homogeneous region.

The process of vortex pairing is shown in Figure 3 where we can see that two vortices rotate each other and merge into one vortex finally. 


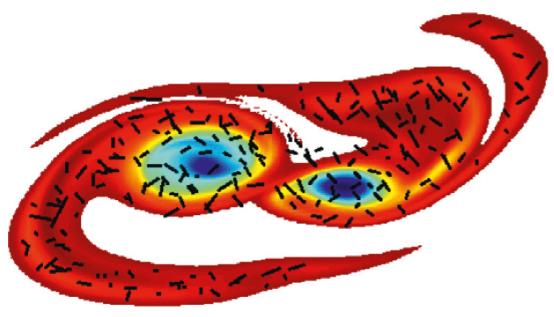

(a)

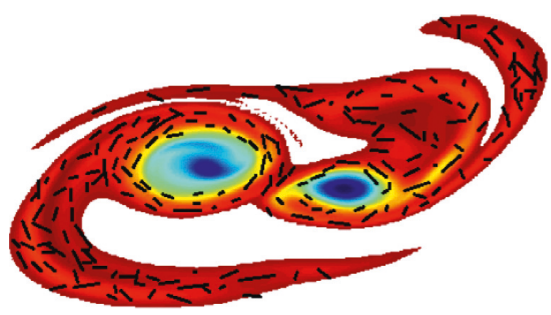

(b)

Figure 4: Particle distribution for different Stokes numbers $(t=100, \mathrm{Re}=200$, and $a=15)$ : (a) St $=0.02$ and (b) St $=1.0$.

4.2. Particle Distribution. The mixing of cylindrical particles can be described by the particle spatial distributions. The rollup of the mixing layer drives the particles to the edge of the vortex by the inertial centrifugal force, and at the same time it brings about an asymmetric shear rate distribution around the particle and generates a normal stress imbalance on the particle surface. The cylindrical particle migration and mixing are dependent on the above two factors.

The mixing degree of the particles is negatively correlated with the inhomogeneous degree of particle distribution. Therefore, the inhomogeneous degree of particle distribution $M_{n}$ is used in order to quantitatively describe the mixing behavior:

$$
M_{n}(t)=\frac{\sum_{i=1}^{N} \sqrt{\left(x_{\mathrm{p} i}(t)-x_{\mathrm{c}}\right)^{2}+\left(y_{\mathrm{p} i}(t)-y_{\mathrm{c}}\right)^{2}}}{\sum_{i=1}^{N} \sqrt{\left(x_{\mathrm{p} i}(0)-x_{\mathrm{c}}\right)^{2}+\left(y_{\mathrm{p} i}(0)-y_{\mathrm{c}}\right)^{2}}},
$$

where $\left(x_{\mathrm{p} i}, y_{\mathrm{p} i}\right)$ and $\left(x_{\mathrm{c}}, y_{\mathrm{c}}\right)$ are the coordinates of the center of the $i$ th particle and vortex center, respectively. A larger value of $M_{n}$ is corresponding to a poorer mixing.

\subsubsection{Effect of the Stokes Number on the Mixing Degree. Figure 4} shows the particle distribution at $t=100$ for St $=0.02$ and 1 . Stokes number $(\mathrm{St})$ is directly related to the function of particle inertia. In the process of rollup of the mixing layer, the cylindrical particles for $\mathrm{St}=0.02$ almost follow fluid streamlines and distribute more homogeneously and are mixed thoroughly. On the contrary, the particles for $\mathrm{St}=1.0$ are centrifugalized from the vortex core to the edge by inertia and are poorly mixed.

Figure 5 shows the change of inhomogeneous degree with time at different Stokes numbers. We can see well mixed for $\mathrm{St}=0.02$. The mixing degree becomes worse with the increase of the Stokes number because more particles are centrifugalized to the edge of the vortex when St is larger.

4.2.2. Effect of the Particle Aspect Ratio on the Mixing Degree. Figure 6 shows the change of inhomogeneous degree with time for different particle aspect ratios. The mixing degree becomes worse as the particle aspect ratio increases because the angular acceleration of the particles is inversely proportional to the particle aspect ratio. Particle angular acceleration and translational motion induced by the fluid

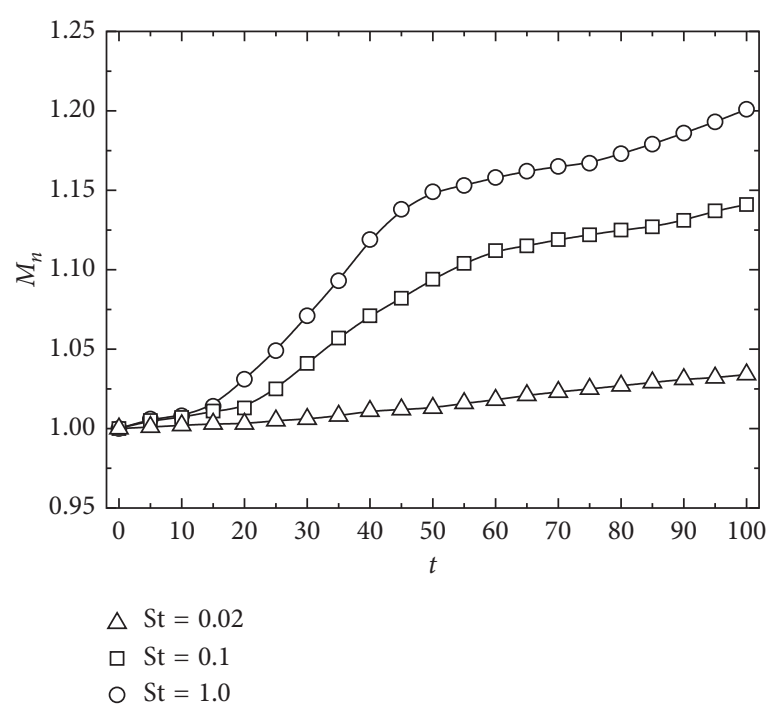

FIgURE 5: Change of inhomogeneous degree with time at different St $(\operatorname{Re}=200$ and $a=15)$.

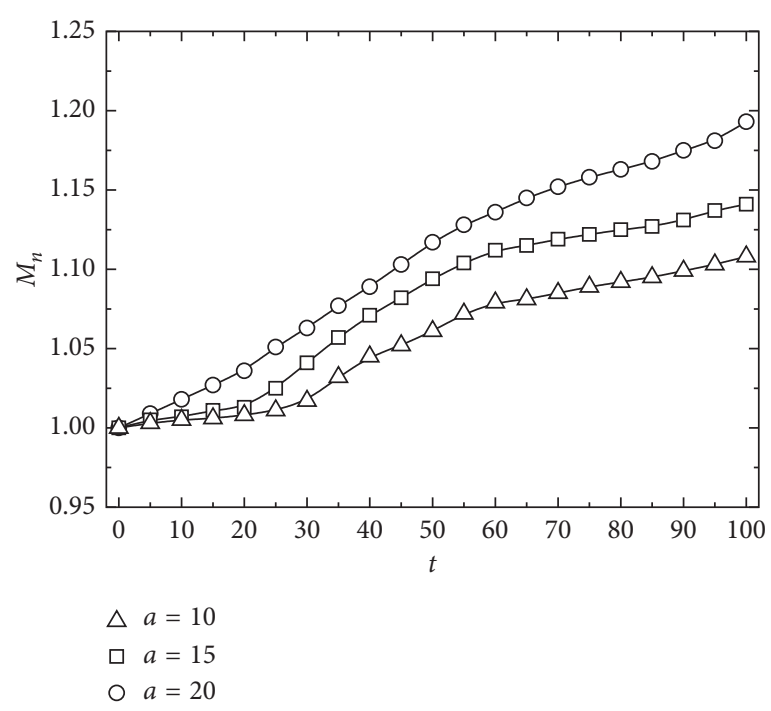

FIGURE 6: Change of inhomogeneous degree with time at different $a(\operatorname{Re}=200$ and $\mathrm{St}=0.1)$.

are smaller for particles with larger particle aspect ratios so that the centrifugal force drives the particles towards the edge of the vortex. 
4.3. Orientation Distribution of the Cylindrical Particle. The cylindrical particles will change the orientation angles $\varphi$ and $\theta$ (shown in Figure 1) under two torques. One torque makes the particle rotate around the vorticity axis, and another torque leads to the particle spin around the flow direction. For describing the orientation distribution, the orientation distribution function $\psi$ is used:

$$
\psi\left(\theta_{i+1}-\theta_{i}\right)=\frac{N_{i+1-i}}{N}
$$

where $\theta$ is shown in Figure 1, $N$ is the total particle number, and $N_{i+1-i}$ is the number of particles with the angles ranging from $i$ to $i+1$.

4.3.1. Effect of the Stokes Number on the Particle Orientation. Figure 7 shows the orientation distribution function of particles for different St. We can see that, with the decrease of the Stokes number, more particles are oriented with the flow direction. This may be attributed that the angular acceleration of the particles is inversely proportional to the Stokes number, and the angular acceleration induced by the fluid stress is larger at smaller Stokes number so that the particles change their orientation more rapidly.

4.3.2. Effect of the Particle Aspect Ratio on the Particle Orientation. The orientation distribution functions of cylindrical particles for different particle aspect ratios are shown in Figure 8. The particle would rotate around its axis of revolution aligned to the vorticity direction when the shear rate is low, while aligning on the flow-gradient plane beyond a critical shear rate value. From the figure, we can see that more particles are oriented on the flow-gradient plane $\left(\theta=0^{\circ}\right)$ and more particles are oriented towards the flow direction with the decrease of the particle aspect ratio.

\section{Conclusion}

The effect of the Stokes number and particle aspect ratio on the mixing and orientation distribution of cylindrical particles in a mixing layer is numerically studied. The mixing of cylindrical particles can be described by the particle spatial distributions. The rollup of the mixing layer drives the particles to the edge of the vortex by the inertial centrifugal force and at the same time brings about an asymmetric shear rate distribution around the particle. The cylindrical particles with the small Stokes number almost follow fluid streamlines and are mixed thoroughly. On the contrary, the particles with the large Stokes number are centrifugalized to the edge of the vortex and are poorly mixed. The mixing degree of particles becomes worse as the particle aspect ratio increases. Particle angular acceleration and translational motion are smaller for that particle with a larger particle aspect ratio so that the centrifugal force drives the particles towards the edge of the vortex. The cylindrical particles will change the orientation angle under the two torques with which the particles would rotate around its axis of revolution aligned to the vorticity direction when the shear rate is low,

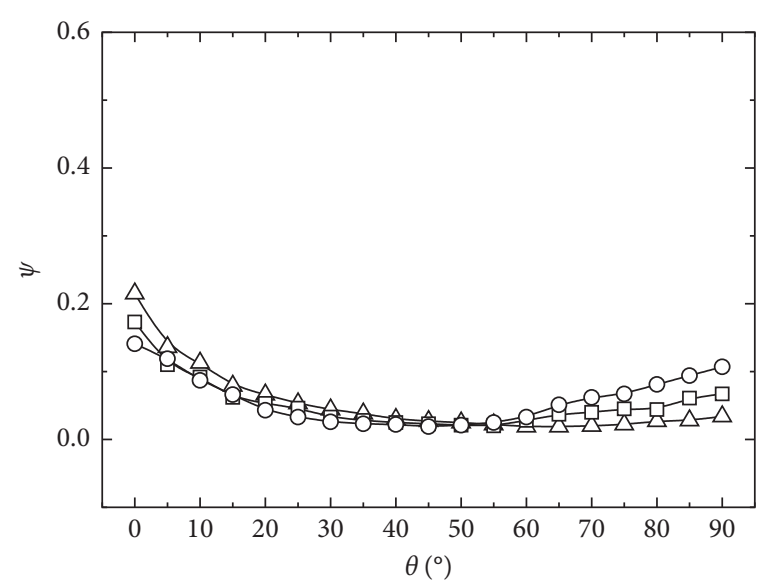

$$
\begin{aligned}
& \triangle \mathrm{St}=0.02 \\
& \square \mathrm{St}=0.1 \\
& \bigcirc \mathrm{St}=1.0
\end{aligned}
$$

FIgURE 7: Orientation distribution of cylindrical particles for different St $(\operatorname{Re}=200$ and $a=15)$.

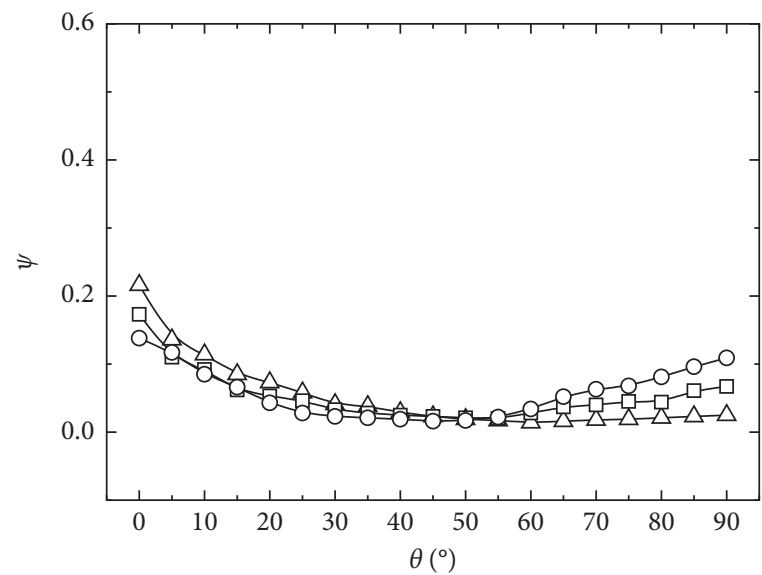

$$
\begin{aligned}
& \triangle a=10 \\
& \square a=15 \\
& \triangle a=20
\end{aligned}
$$

Figure 8: Orientation distribution of cylindrical particles for different $a(\mathrm{Re}=200$ and $\mathrm{St}=0.1)$.

while aligning on the flow-gradient plane beyond a critical shear rate value. More particles are oriented with the flow direction, and this phenomenon becomes more obvious with the decrease of the Stokes number and particle aspect ratio.

\section{Data Availability}

The data used to support the findings of this study are available from the corresponding author upon request.

\section{Conflicts of Interest}

The authors declare that there are no conflicts of interest regarding the publication of this paper. 


\section{Acknowledgments}

The authors would like to thank the Major Program of National Natural Science Foundation of China (Grant no. 11631216).

\section{References}

[1] V. N. Singh, B. R. Mehta, R. K. Joshi, and F. E. Kruis, "Effect of silver addition on the ethanol-sensing properties of indium oxide nanoparticle layers: optical absorption study," Journal of Nanomaterials, vol. 2007, Article ID 28031, 5 pages, 2007.

[2] I. Aruna, F. E. Kruis, S. Kundu, M. Muhler, R. Theissmann, and M. Spasova, "CO ppb sensors based on monodispersed SnOx : Pd mixed nanoparticle layers: insight into dual conductance response," Journal of Applied Physics, vol. 105, no. 6, p. 064312, 2009.

[3] F. W. Hsiao, Y. L. Lee, and C. H. Chang, "Formation and characterization of mixed polyelectrolyte-surfactant Langmuir layer templates for silver nanoparticle growth," Colloids and Surfaces A: Physicochemical and Engineering Aspects, vol. 351, no. 1-3, pp. 18-25, 2009.

[4] L. Liggieri, E. Santini, E. Guzman, A. Maestro, and F. Ravera, "Wide-frequency dilational rheology investigation of mixed silica nanoparticle-CTAB interfacial layers," Soft Matter, vol. 7, no. 17, pp. 7699-7709, 2011.

[5] M. L. Xie, M. Z. Yu, and L. P. Wang, "A TEMOM model to simulate nanoparticle growth in the temporal mixing layer due to Brownian coagulation," Journal of Aerosol Science, vol. 54, pp. 32-48, 2012.

[6] P. A. Yazhgur, B. A. Noskov, L. Liggieri et al., "Dynamic properties of mixed nanoparticle/surfactant adsorption layers," Soft Matter, vol. 9, no. 12, pp. 3305-3314, 2013.

[7] K. H. Kim, H. M. An, H. D. Kim, and T. G. Kim, "Transparent conductive oxide films mixed with gallium oxide nanoparticle/single-walled carbon nanotube layer for deep ultraviolet light-emitting diodes," Nanoscale Research Letters, vol. 8, no. 1, pp. 507-518, 2013.

[8] N. J. Murfield and S. C. Garrick, "Large eddy simulation and direct numerical simulation of homogeneous nucleation in turbulent wakes," Journal of Aerosol Science, vol. 60, pp. 21-33, 2013.

[9] A. J. Fager and S. C. Garrick, "Metal particle nucleation in turbulent jets visualization of the large and small scales," Journal of Visualization, vol. 16, no. 4, pp. 297-302, 2013.

[10] E. Guzman, E. Santini, M. Ferrari, L. Liggieri, and F. Ravera, "Interfacial properties of mixed DPPC-hydrophobic fumed silica nanoparticle layers," Journal of Physical Chemistry C, vol. 119, no. 36, pp. 21024-21034, 2015.

[11] D. Orsi, E. Guzman, L. Liggieri et al., "2D dynamical arrest transition in a mixed nanoparticle-phospholipid layer studied in real and momentum spaces," Scientific Reports, vol. 5, no. 1, p. 17930, 2015.

[12] S. C. Garrick, "Growth mechanisms of nanostructured titania in turbulent reacting flows," Journal of Nanotechnology, vol. 2015, Article ID 642014, 10 pages, 2015.

[13] S. Kerli and U. Alver, "Preparation and characterization of $\mathrm{ZnO} / \mathrm{NiO}$ nanocomposite particles for solar cell applications," Journal of Nanotechnology, vol. 2016, Article ID 4028062, 5 pages, 2016.

[14] A. Michalke, "On the inviscid instability of the hyperbolictangent velocity profile," Journal of Fluid Mechanics, vol. 19, pp. 543-556, 1964. 


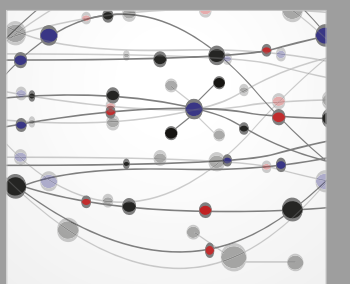

The Scientific World Journal
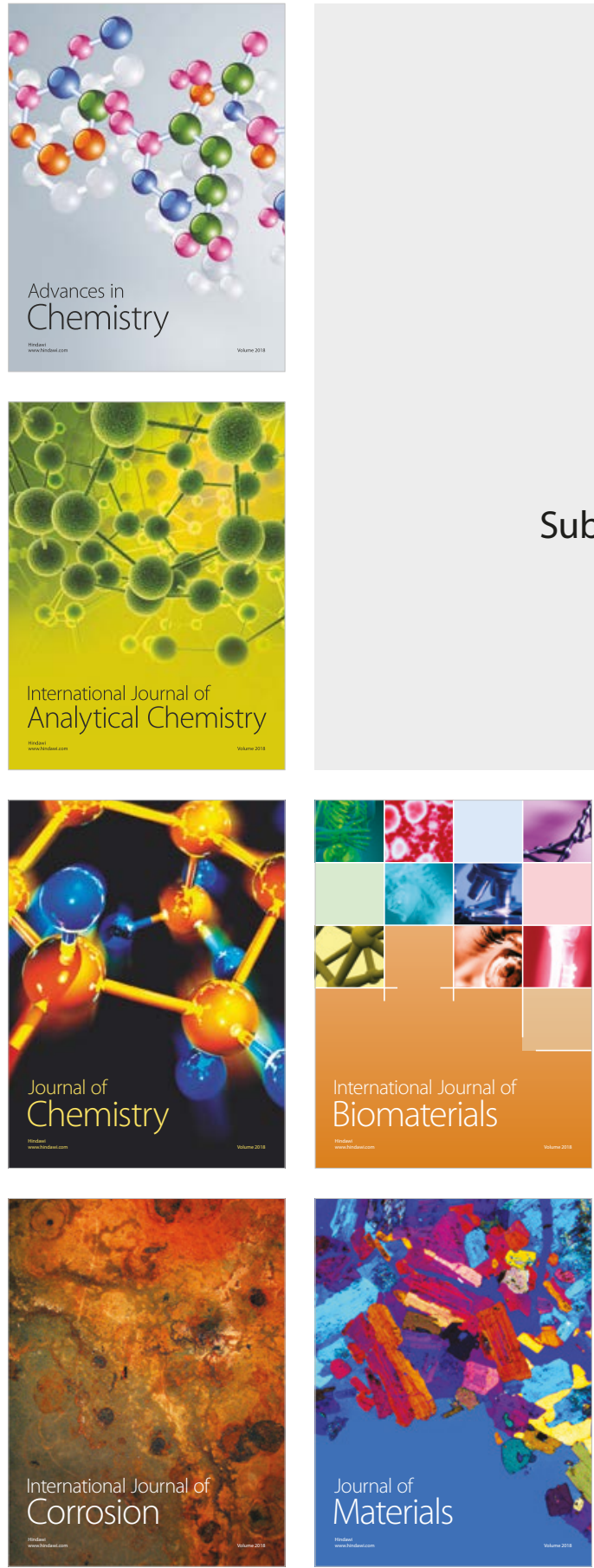

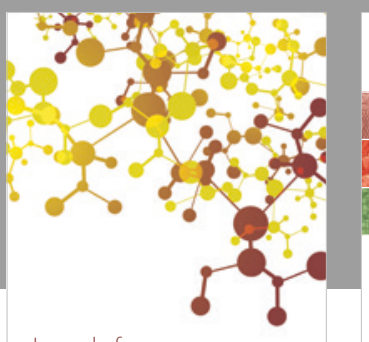

Journal of

Applied Chemistry
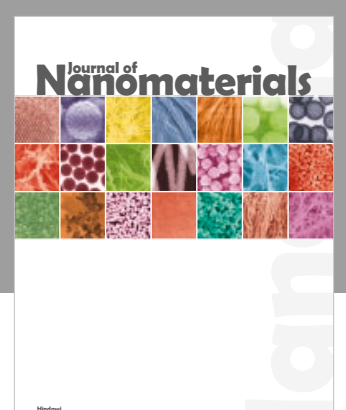

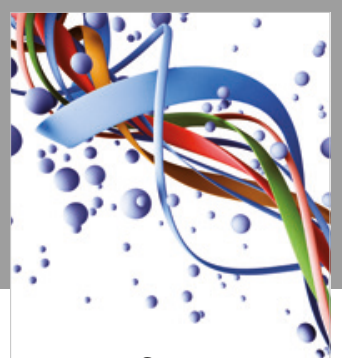

Scientifica

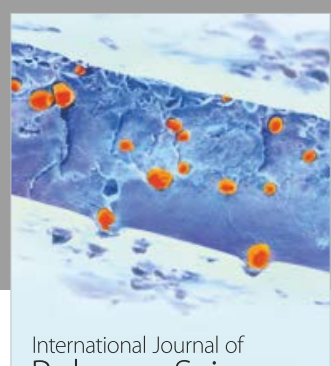

Polymer Science

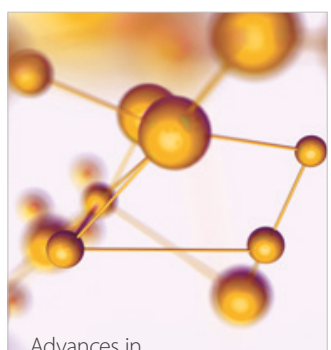

Physical Chemistry
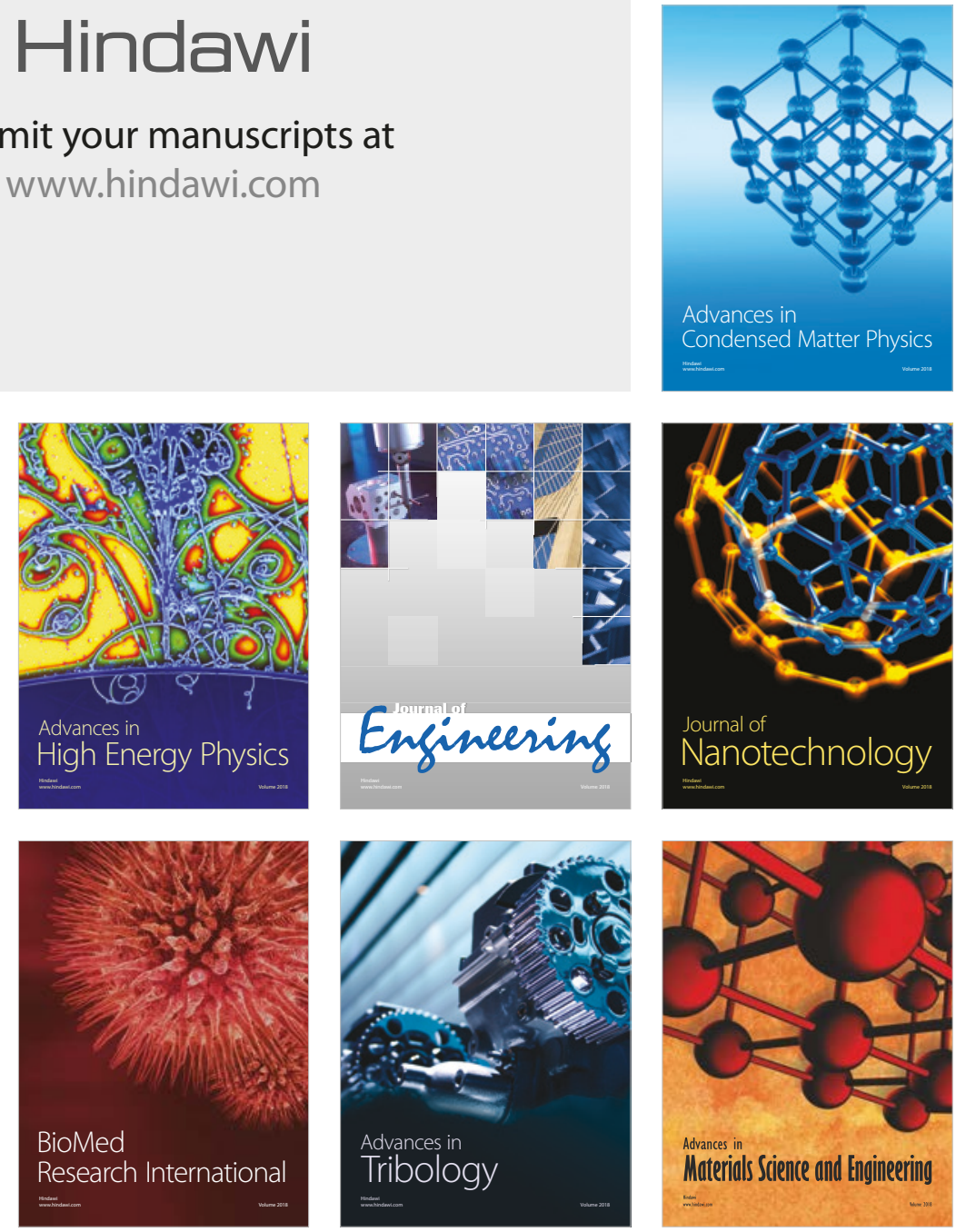